\title{
Propuesta de evaluación de las competencias de comunicación oral y escrita en asignaturas de Ingeniería Mecánica y Ciencia de Materiales
}

\author{
O. Sahuquillo ${ }^{\mathrm{a}}$, A. Sonsecab ${ }^{\mathrm{b}}$ J. Martínez ${ }^{\mathrm{c}}$, J. Carballeira ${ }^{\mathrm{d}}$, F.D. Denia ${ }^{\mathrm{e}}$ \\ J.J. Ródenas ${ }^{\mathrm{f}}$ O. Marco ${ }^{\mathrm{g}}$
}

Departamento de Ingeniería Mecánica y de Materiales Universitat Politècnica de València, Camino de Vera s/n, 46022, Valencia
email: aossana@upvnet.upv.es, bagsonol@posgrado.upv.es,_ cjomarc12@mcm.upv.es, djacarmo@mcm.upv.es, efdenia@mcm.upv.es, fjjrodena@mcm.upv.es,
gonmaral@upvnet.upv.es

\begin{abstract}
Resumen
La acreditación internacional de los másteres y grados ofertados por la universidad y la actual exigencia de los contratantes, han llevado a la necesidad de especificar en los planes de estudio, qué se ha estudiado (contenido) y las competencias adquiridas. En los últimos años el enfoque basado en competencias del Espacio Europeo de Educación Superior se ha utilizado en el desarrollo de los nuevos planes de estudios, la evaluación de estas competencias es todavía una tarea pendiente. En este trabajo se presenta una propuesta para la evaluación de la competencia de comunicación, tanto oral como escrita, en asignaturas de Ingeniería Mecánica y Ciencia de Materiales. La evaluación de las competencias en los resultados de aprendizaje se realizó a partir del desarrollo de rúbricas con el objetivo de cuantificar el nivel de consecución. Las rúbricas han permitido observar variaciones en el nivel de la competencia entre alumnos de Máster y de Grado, además de una buena correlación entre las valoraciones de los profesores evaluadores de un mismo acto de evaluación. Se completarán los resultados con una encuesta planteada a los alumnos donde se pretende recoger su valoración sobre la dinámica planteada.
\end{abstract}

Palabras clave: competencias genéricas, comunicación eficaz, resultados de aprendizaje, feedback 
Propuesta de evaluación de las competencias de comunicación oral y escrita en asignaturas de Ingeniería Mecánica y Ciencia de Materiales

\section{Introducción}

Dentro del marco del Espacio Europeo de Educación Superior los títulos de Grado y de Máster desarrollados por la universidad siguen un enfoque basado en competencias (Sursock, 2010; Murias, 2007; Rieckmann, 2012).

En estos programas se definen claramente las competencias genéricas y específicas de cada grado, así como las asignaturas donde se trabajarán durante la titulación. La evaluación de las competencias específicas y su grado de logro continúa reflejándose en los planes de estudio con calificaciones numéricas, mientras que la evaluación de la competencias genéricas es una tarea pendiente y por definir. Se entiende que los estudiantes adquieren estas capacidades y habilidades al final de su formación en la titulación. De otro lado, la acreditación internacional de estos programas, junto con la necesidad de disponer de una información útil sobre las competencias de los alumnos por parte del mercado de trabajo y de los encargados de buscar empleados, han hecho que la universidad se ponga a trabajar en este sentido (Andrews, 2008; Entwistle, 2004).

Este trabajo presenta algunos resultados obtenidos en el marco de un proyecto de innovación educativa (programa PIME) en la evaluación de tres competencias genéricas que se han trabajado tradicionalmente en asignaturas de ingeniería mecánica y ciencia de materiales: capacidad de análisis de problemas, capacidad de aplicación del pensamiento práctico y habilidades de comunicación oral y escrita (Tuning (2014), http://www.unideusto.org/tuningeu). En particular, en este trabajo se han desarrollado metodologías para el análisis de las habilidades en comunicación oral y escrita (Sparks, 2014; Dunbar, 2006). Se busca obtener información con ciertas garantías de los puntos débiles y de aquellos con mayor dominio por parte de los estudiantes en las competencias de comunicación (Jonsson, 2007). Por otra parte, estas metodologías de evaluación tratan de motivar la participación de los alumnos y aumentar su interés durante las clases. La tarea se centra en explicar oralmente a sus compañeros la información más relevante de la sesión anterior (competencia comunicación oral). En el caso de la competencia escrita se desarrollará un informe técnico concerniente a la resolución de un problema.

\section{Objetivos}

El presente trabajo consiste en una propuesta para la evaluación de las competencias genéricas, sin obviar las dificultades circundantes a este tema. Se centra en la evaluación de la competencia de comunicación eficaz tanto a nivel oral como escrito. El objetivo general es obtener información fiable para evaluar fortalezas y debilidades de los estudiantes en la competencia de comunicación. En este sentido, se plantean los siguientes objetivos específicos: 
- Desarrollar herramientas de evaluación para los resultados de aprendizaje relacionados con las competencias objeto de evaluación.

- Implementar la metodología y valorar los resultados de aprendizaje.

- Valorar las competencias en diferentes niveles formativos: diferentes cursos y titulaciones.

- Análizar los resultados: valoración alumno-alumno, profesor-alumno y profesorprofesor.

- Elaborar una encuesta donde recoger la opinión de los alumnos sobre la metodología utilizada, obteniendo el feedback.

\section{Desarrollo de la innovación}

El desarrollo de la propuesta se llevó a cabo en dos asignaturas: Ciencia de Materiales del Grado en Ingeniería Química y Ampliación de Vibraciones del Máster de Ingeniería Aeronáutica, ambas asignaturas ofertadas en titulaciones de la Universitat Politècnica de València.

En el caso de la competencia en comunicación oral, en la asignatura de grado, los alumnos se dividirán en grupos de 4-5 estudiantes y en la asignatura de máster de forma individual. El trabajo de los alumnos se desarrolla en el periodo comprendido entre dos sesiones sucesivas, aproximadamente cada dos semanas para maximizar las posibilidades de autoaprendizaje, debido a que expondrán un resumen de la sesión anterior a los compañeros. Con esta premisa, se les requiere la preparación de una presentación oral, 10-15 minutos, con la ayuda del documento preparado que podrán visualizar durante el desarrollo de la exposición, y llevarán a cabo la explicación al resto de compañeros de los aspectos de la sesión anterior que consideren más importantes. Para asegurar la preparación de la tarea de todos los alumnos, el profesor elegirá de forma aleatoria al estudiante que realizará la exposición. Se espera que los estudiantes interaccionen ente ellos, compartan conceptos, planteen cuestiones a sus compañeros y desarrollen estrategias que los hagan responsables de su propio aprendizaje considerando que serán evaluados también por el resto de estudiantes. Se pretende que los alumnos, además de trabajar la habilidad de la expresión oral, mejoren el aprendizaje autónomo, la capacidad de síntesis, el pensamiento crítico y la responsabilidad en la búsqueda de información, análisis y verificación de la información. La evaluación de la exposición oral realizada se llevará a cabo con la rúbrica desarrollada a tal efecto, ver Tabla 1, obteniendo valoración de dos profesores y del resto de compañeros, haciéndoles de este modo partícipes de la evaluación. Adicionalmente, el profesor en base a la exposición realizada corregirá los errores o aclarará aspectos que se detecten como confusos relacionados con la materia, reportando sobre todos los alumnos feedback, reforzando de este modo lo visto y trabajado en la sesión anterior. 
Propuesta de evaluación de las competencias de comunicación oral y escrita en asignaturas de Ingeniería Mecánica y Ciencia de Materiales

Tabla 1. Rúbrica expresión oral

\begin{tabular}{|c|c|c|c|c|c|c|c|}
\hline & CRITERIOS & 0-No cumple & 1-Deficiente & 2-Regular & 3-Bueno & 4-Muy bueno & 5-Excelente \\
\hline 1 & $\begin{array}{l}\text { Realiza una } \\
\text { introducción } \\
\text { efectiva al tema }\end{array}$ & no la realiza & $\begin{array}{l}\text { realiza } \\
\text { introducción } \\
\text { mayoritariamente } \\
\text { incompleta }\end{array}$ & $\begin{array}{l}\text { realiza } \\
\text { introducción } \\
\text { incompleta }\end{array}$ & \begin{tabular}{|l} 
realiza \\
introducción
\end{tabular} & $\begin{array}{l}\text { realiza } \\
\text { introducción y } \\
\text { pone en situación } \\
\text { a la audiencia }\end{array}$ & $\begin{array}{l}\text { realiza } \\
\text { introducción } \\
\text { indicando } \\
\text { ejemplos del } \\
\text { interés del tema }\end{array}$ \\
\hline 2 & $\begin{array}{l}\text { Señala los objetivos } \\
\text { e ideas principales }\end{array}$ & no lo realiza & $\begin{array}{l}\text { señala alguno de } \\
\text { los objetivos }\end{array}$ & $\begin{array}{l}\text { señala los } \\
\text { objetivos de forma } \\
\text { incompleta }\end{array}$ & $\begin{array}{l}\text { señala todos los } \\
\text { objetivos }\end{array}$ & $\begin{array}{l}\text { señala todos los } \\
\text { objetivos de forma } \\
\text { organizada y } \\
\text { sintética }\end{array}$ & $\begin{array}{l}\text { señala todos los } \\
\text { objetivos de forma } \\
\text { organizada y } \\
\text { sintética, con un } \\
\text { enfoque aplicado }\end{array}$ \\
\hline 3 & $\begin{array}{l}\text { Presenta resultados } \\
\text { fundamentados } \\
\text { adecuadamente }\end{array}$ & no presenta & $\begin{array}{l}\text { presenta algún } \\
\text { resultado }\end{array}$ & $\begin{array}{l}\text { presenta de forma } \\
\text { incompleta }\end{array}$ & $\begin{array}{l}\text { presenta todos los } \\
\text { resultados }\end{array}$ & $\begin{array}{l}\text { presenta los } \\
\text { resultados } \\
\text { relevantes }\end{array}$ & $\begin{array}{l}\text { presenta los } \\
\text { resultados } \\
\text { relevantes y los } \\
\text { fundamenta }\end{array}$ \\
\hline 4 & $\begin{array}{l}\text { Conclusiones } \\
\text { apropiadas y } \\
\text { sintéticas }\end{array}$ & no las realiza & $\begin{array}{l}\text { señala alguna } \\
\text { conclusión }\end{array}$ & $\begin{array}{l}\text { señala } \\
\text { conclusiones de } \\
\text { forma incompleta }\end{array}$ & $\begin{array}{l}\text { señala todas las } \\
\text { conclusiones }\end{array}$ & $\begin{array}{l}\text { señala las } \\
\text { conclusiones más } \\
\text { relevantes } \\
\text { organizaday } \\
\text { sintéticamente }\end{array}$ & $\begin{array}{l}\text { señala las } \\
\text { conclusiones más } \\
\text { relevantes de } \\
\text { forma organizada, } \\
\text { sintética y } \\
\text { evalúa/analiza sus } \\
\text { consecuencias }\end{array}$ \\
\hline 5 & $\begin{array}{l}\text { Interpreta, } \\
\text { argumenta y } \\
\text { justifica la } \\
\text { información } \\
\text { presentada }\end{array}$ & no lo realiza & $\begin{array}{l}\text { Lo realiza de } \\
\text { manera errónea }\end{array}$ & $\begin{array}{l}\text { Lo realiza bien } \\
\text { pero de manera } \\
\text { muy limitada }\end{array}$ & $\begin{array}{l}\text { Lo realiza bien } \\
\text { pero incompleto }\end{array}$ & $\begin{array}{l}\text { Lo realiza de } \\
\text { manera correcta }\end{array}$ & $\begin{array}{l}\text { Lo realiza } \\
\text { correctamente, y } \\
\text { enfatizando los } \\
\text { aspectos } \\
\text { relevantes }\end{array}$ \\
\hline 6 & $\begin{array}{l}\text { Presentación } \\
\text { estructurada, clara, } \\
\text { coherente y eficaz }\end{array}$ & no cumple ninguna & $\begin{array}{l}\text { parcialmente } \\
\text { estructurada }\end{array}$ & es estructurada & $\begin{array}{l}\text { al menos es } \\
\text { estructurada y } \\
\text { clara }\end{array}$ & $\begin{array}{l}\text { al menos es } \\
\text { estructurada, clara } \\
\text { y coherente }\end{array}$ & $\begin{array}{l}\text { al menos es } \\
\text { estructurada, } \\
\text { clara, coherente y } \\
\text { eficaz }\end{array}$ \\
\hline 7 & $\begin{array}{l}\text { Utiliza un lenguaje } \\
\text { técnico adecuado }\end{array}$ & nunca & $\begin{array}{l}\text { lo usa con errores } \\
\text { reiterados }\end{array}$ & $\begin{array}{l}\text { lo usa con algún } \\
\text { error }\end{array}$ & $\begin{array}{l}\text { lo utiliza } \\
\text { ocasionalmente }\end{array}$ & $\begin{array}{l}\text { lo utiliza habitual y } \\
\text { adecuadamente }\end{array}$ & $\begin{array}{l}\text { lo utiliza habitual y } \\
\text { adecuadamente, e } \\
\text { introduce nuevos } \\
\text { conceptos }\end{array}$ \\
\hline 8 & $\begin{array}{l}\text { Utiliza los recursos } \\
\text { disponibles para una } \\
\text { comunicación más } \\
\text { eficiente }\end{array}$ & $\begin{array}{l}\text { no utiliza los } \\
\text { recursos } \\
\text { disponibles }\end{array}$ & $\begin{array}{l}\text { utiliza los recursos } \\
\text { de forma } \\
\text { inadecuada }\end{array}$ & $\begin{array}{l}\text { utiliza los recursos } \\
\text { pero no para } \\
\text { clarificar ideas }\end{array}$ & $\begin{array}{l}\text { utiliza los recursos } \\
\text { para clarificar las } \\
\text { ideas principales }\end{array}$ & $\begin{array}{l}\text { utiliza los recursos } \\
\text { para clarificar las } \\
\text { ideas de forma } \\
\text { generalizada }\end{array}$ & $\begin{array}{l}\text { utiliza los recursos } \\
\text { disponiblese e } \\
\text { introduce nuevos } \\
\text { para clarificar las } \\
\text { ideas de forma } \\
\text { generalizada }\end{array}$ \\
\hline 9 & $\begin{array}{l}\text { La presentación se } \\
\text { ajusta al tiempo } \\
\text { disponible }\end{array}$ & $\begin{array}{l}\text { Ningún control } \\
\text { temporal de la } \\
\text { extensión parcial y } \\
\text { total }\end{array}$ & $\begin{array}{l}\text { Se excede o le } \\
\text { sobra demasiado } \\
\text { tiempo }\end{array}$ & $\begin{array}{l}\text { Se ajusta al tiempo } \\
\text { disponible de } \\
\text { forma aproximada }\end{array}$ & $\begin{array}{l}\text { Se ajusta al tiempo } \\
\text { disponible }\end{array}$ & $\begin{array}{l}\text { Se ajusta al tiempo } \\
\text { disponible, dedica } \\
\text { el tiempo } \\
\text { apropiado en cada } \\
\text { parte }\end{array}$ & $\begin{array}{l}\text { Se ajusta al tiempo } \\
\text { disponible, dedica } \\
\text { el tiempo } \\
\text { apropiado en cada } \\
\text { parte y se } \\
\text { reorganiza en caso } \\
\text { necesario }\end{array}$ \\
\hline 10 & $\begin{array}{l}\text { Dicción clara, sin } \\
\text { muletillas, tono } \\
\text { adecuado, postura } \\
\text { corporal adecuada y } \\
\text { contacto visual }\end{array}$ & no cumple ninguna & cumple 1 & $\begin{array}{l}\text { Dicción clara y } \\
\text { tono monótono }\end{array}$ & $\begin{array}{l}\text { Dicción clara y } \\
\text { postura corporal }\end{array}$ & $\begin{array}{l}\text { Dicción clara, } \\
\text { postura corporal y } \\
\text { tono adecuado }\end{array}$ & $\begin{array}{l}\text { Dicción clara, } \\
\text { postura corporal, } \\
\text { tono adecuado y } \\
\text { contacto visual }\end{array}$ \\
\hline 11 & $\begin{array}{l}\text { Analiza, valora y } \\
\text { responde a las } \\
\text { preguntas que se } \\
\text { formulan }\end{array}$ & $\begin{array}{l}\text { No cumple } \\
\text { ninguna }\end{array}$ & $\begin{array}{l}\text { Analiza, pero ni } \\
\text { valora ni responde }\end{array}$ & $\begin{array}{l}\text { Analiza y valora, } \\
\text { pero no responde }\end{array}$ & $\begin{array}{l}\text { Analiza, valora y } \\
\text { responde de } \\
\text { manera limitada, } \\
\text { con ayuda del } \\
\text { profesor }\end{array}$ & $\begin{array}{l}\text { Analiza, valora y } \\
\text { responde con } \\
\text { enfoque propio }\end{array}$ & $\begin{array}{l}\text { Analiza, valora y } \\
\text { responde con } \\
\text { enfoque propio, y } \\
\text { plantea cuestiones } \\
\text { relacionadas }\end{array}$ \\
\hline
\end{tabular}

Las presentaciones orales de los alumnos fueron evaluadas considerando once ítems diferentes. Los cuatro primeros principalmente relacionados con la organización, la estructura y el contenido de la presentación, es decir, que corresponden a la misma dimensión (contenido). El quinto punto está enfocado a valorar la eficacia en la interpretación y justificación de la información presentada y el sexto evalúa la claridad en la estructura y en

(c)) EY-NC-ND 2015, Universitat Politècnica de València 
la comunicación del tema a la audiencia por parte del alumno. El séptimo punto se valora la utilización del lenguaje técnico, y el octavo se relaciona con el uso de recursos adicionales (pizarra, objetos, ejemplos ...) que garanticen una mayor eficacia en la presentación de la información a la audiencia. Los puntos noveno y décimo estan relacionados con la planificación temporal y la corrección del orador en términos de dicción, posición corporal, tono de voz y contacto visual. Por último, para evaluar la capacidad de análisis y de respuesta, profesores y/o estudiantes, hicieron preguntas para valorar este ítem.

En el caso de la competencia en expresión escrita, los alumnos trabajarán de forma individual y se evaluará únicamente en los alumnos de máster. Deberán desarrollar dos casos de estudio abiertos. Se pretende que se involucren en un problema próximo a la realidad en el que aplicar el conocimiento adquirido durante el curso, decidiendo la estrategia, el método a aplicar y la toma de decisiones para solucionar el problema, explicando la secuencia seguida para alcanzar la solución por medio de un informe que demuestre la aplicación de conocimientos adquiridos durante la asignatura. Además del desarrollo de la competencia escrita, ofrece a los estudiantes un método de aprendizaje activo, así como un desafío y una oportunidad de trabajo con un problema real, donde plantear hipótesis para encontrar la mejor solución y poner en práctica el conocimiento individual adquirido. Para la valoración de los informes elaborados por los alumnos se ha desarrollado una rúbrica para determinar el nivel de consecución de la competencia comunicación escrita, ver Tabla 2.

Al igual que en la evaluación de comunicación oral, los informes escritos de los estudiantes se evaluaron a través de diez puntos. Los cuatro primeros relacionados con el contenido (introducción, objetivos, resultados y conclusiones). El quinto ítem se centra en la estructura y coherencia formal de la información, mientras que el sexto evalúa la calidad en términos de estilo y formato del informe. El séptimo se correlaciona con la presencia de errores gramaticales y ortográficos, y el octavo valora el uso apropiado del lenguaje técnico. El noveno y el décimo evalúan el logro en la realización de un informe profesional y de calidad a través de la argumentación y la justificación de la información presentada, así como mediante el uso de recursos gráficos para resaltar los resultados más relevantes. 
Propuesta de evaluación de las competencias de comunicación oral y escrita en asignaturas de Ingeniería Mecánica y Ciencia de Materiales

Tabla 2. Rúbrica expresión escrita

\begin{tabular}{|c|c|c|c|c|c|c|c|}
\hline & & 0-No cumple & 1-Deficiente & 2-Regular & 3-Bueno & 4-Muy bueno & 5-Excelente \\
\hline & CRITERIOS & & & & & & \\
\hline 1 & $\begin{array}{l}\text { Realiza una } \\
\text { introducción } \\
\text { efectiva al tema }\end{array}$ & No la realiza & $\begin{array}{l}\text { Realiza introducción } \\
\text { mayoritariamente } \\
\text { incompleta }\end{array}$ & $\begin{array}{l}\text { Realiza introducción } \\
\text { incompleta }\end{array}$ & Realiza introducción & $\begin{array}{l}\text { Realiza introducción } \\
\text { y pone en situación } \\
\text { a los lectores }\end{array}$ & $\begin{array}{l}\text { Realiza introducción } \\
\text { indicando ejemplos } \\
\text { del interés del tema }\end{array}$ \\
\hline 2 & $\begin{array}{l}\text { Identifica los } \\
\text { objetivose ideas } \\
\text { principales }\end{array}$ & No lo realiza & $\begin{array}{l}\text { Señala alguno de los } \\
\text { objetivos }\end{array}$ & $\begin{array}{l}\text { Seh́ala los objetivos } \\
\text { de forma } \\
\text { incompleta }\end{array}$ & $\begin{array}{l}\text { Seĥala todos los } \\
\text { objetivos }\end{array}$ & $\begin{array}{l}\text { Sef̂ala todos los } \\
\text { objetivos de forma } \\
\text { organizada y } \\
\text { sintética }\end{array}$ & $\begin{array}{l}\text { Señala todos los } \\
\text { objetivos de forma } \\
\text { organizada y } \\
\text { sintética, } \\
\text { relacionándolos con } \\
\text { situaciones reales }\end{array}$ \\
\hline 3 & $\begin{array}{l}\text { Los resultados se } \\
\text { presentan } \\
\text { fundamentados } \\
\text { adecuadamente }\end{array}$ & No presenta & $\begin{array}{l}\text { Presenta algún } \\
\text { resultado }\end{array}$ & $\begin{array}{l}\text { Presenta de forma } \\
\text { incompleta }\end{array}$ & $\begin{array}{l}\text { Presenta todos los } \\
\text { resultados }\end{array}$ & $\begin{array}{l}\text { Presenta los } \\
\text { resultados } \\
\text { relevantes }\end{array}$ & $\begin{array}{l}\text { Presenta los } \\
\text { resultados } \\
\text { relevantes y los } \\
\text { fundamenta }\end{array}$ \\
\hline 4 & $\begin{array}{l}\text { Las conclusiones } \\
\text { son apropiadas y } \\
\text { sintéticas }\end{array}$ & No las realiza & $\begin{array}{l}\text { Señala alguna } \\
\text { conclusión } \\
\text { incompleta }\end{array}$ & $\begin{array}{l}\text { Señala todas las } \\
\text { conclusiones de } \\
\text { forma incompleta }\end{array}$ & $\begin{array}{l}\text { Señala todas las } \\
\text { conclusiones }\end{array}$ & $\begin{array}{l}\text { Señala las } \\
\text { conclusiones más } \\
\text { relevantes de forma } \\
\text { organizada y } \\
\text { sintética }\end{array}$ & $\begin{array}{l}\text { Señala las } \\
\text { conclusiones más } \\
\text { relevantes de forma } \\
\text { organizada, } \\
\text { sintética y } \\
\text { evalúa/analiza sus } \\
\text { consecuencias }\end{array}$ \\
\hline 5 & $\begin{array}{l}\text { Informe } \\
\text { estructurado y } \\
\text { coherente }\end{array}$ & No estructurado & $\begin{array}{l}\text { Estructura } \\
\text { incoherente }\end{array}$ & $\begin{array}{l}\text { Estructura } \\
\text { coherente pero } \\
\text { incompleta }\end{array}$ & $\begin{array}{l}\text { Estructura } \\
\text { coherente }\end{array}$ & $\begin{array}{l}\text { Estructura } \\
\text { coherente, relación } \\
\text { entre secciones }\end{array}$ & $\begin{array}{l}\text { Estructura } \\
\text { coherente, relación } \\
\text { entre seccionesy } \\
\text { orden justificado }\end{array}$ \\
\hline 6 & $\begin{array}{l}\text { Formato y estilo } \\
\text { profesional }\end{array}$ & No lo realiza & $\begin{array}{l}\text { Formato y estilo } \\
\text { inconsistente }\end{array}$ & $\begin{array}{l}\text { Formato y estilo } \\
\text { consistente pero } \\
\text { limitado }\end{array}$ & $\begin{array}{l}\text { Formato y estilo } \\
\text { correcto }\end{array}$ & $\begin{array}{l}\text { Formato y estilo } \\
\text { avanzado }\end{array}$ & $\begin{array}{l}\text { Formato y estilo } \\
\text { profesional }\end{array}$ \\
\hline 7 & Ortografia & $\begin{array}{l}\text { Carencia total de } \\
\text { ortografía }\end{array}$ & $\begin{array}{l}\text { Elevadas faltas de } \\
\text { ortografia }\end{array}$ & $\begin{array}{l}\text { Alguna falta de } \\
\text { ortografía presente }\end{array}$ & $\begin{array}{l}\text { Redacción sin faltas } \\
\text { de ortografía }\end{array}$ & $\begin{array}{l}\text { Ortografía correcta } \\
\text { ytécnica }\end{array}$ & $\begin{array}{l}\text { Ortografía correcta, } \\
\text { técnica y apreciado } \\
\text { uso de sinónimos }\end{array}$ \\
\hline 8 & $\begin{array}{l}\text { Utiliza un lenguaje } \\
\text { técnico adecuado }\end{array}$ & $\begin{array}{l}\text { Lenguaje } \\
\text { inadecuado }\end{array}$ & $\begin{array}{l}\text { Lo usa con errores } \\
\text { reiterados }\end{array}$ & $\begin{array}{l}\text { Lo usa con algún } \\
\text { error }\end{array}$ & $\begin{array}{l}\text { Lo utiliza } \\
\text { ocasionalmente }\end{array}$ & $\begin{array}{l}\text { Lo utiliza habitual y } \\
\text { adecuadamente }\end{array}$ & $\begin{array}{l}\text { Lo utiliza habitual y } \\
\text { adecuadamente, e } \\
\text { introduce nuevos } \\
\text { conceptos }\end{array}$ \\
\hline 9 & $\begin{array}{l}\text { Interpreta, } \\
\text { argumenta y } \\
\text { justifica la } \\
\text { información }\end{array}$ & Nolo realiza & $\begin{array}{l}\text { Lo realiza de } \\
\text { manera errónea }\end{array}$ & $\begin{array}{l}\text { Lo realiza bien pero } \\
\text { de manera muy } \\
\text { limitada }\end{array}$ & $\begin{array}{l}\text { Lo realiza bien pero } \\
\text { aún incompleto }\end{array}$ & $\begin{array}{l}\text { Lo realiza de } \\
\text { manera correcta }\end{array}$ & $\begin{array}{l}\text { Lo realiza } \\
\text { correctamente, y } \\
\text { además con el } \\
\text { énfasis necesario }\end{array}$ \\
\hline 10 & $\begin{array}{l}\text { Elabora y utiliza } \\
\text { recursos gráficos } \\
\text { efectivos que dan } \\
\text { calidad al informe }\end{array}$ & $\begin{array}{l}\text { No utiliza recurso } \\
\text { alguno }\end{array}$ & $\begin{array}{l}\text { Utiliza recursos de } \\
\text { forma inadecuada }\end{array}$ & $\begin{array}{l}\text { Utiliza recursos } \\
\text { pero no aportan } \\
\text { calidad }\end{array}$ & $\begin{array}{l}\text { Utiliza recursos que } \\
\text { aportan calidad, } \\
\text { pero limitados }\end{array}$ & $\begin{array}{l}\text { Utiliza recursos que } \\
\text { aportan calidad }\end{array}$ & $\begin{array}{l}\text { Utiliza recursos } \\
\text { convencionales e } \\
\text { introduce nuevos } \\
\text { que dan } \\
\text { profesionalidad }\end{array}$ \\
\hline
\end{tabular}

Para la evaluación de habilidades orales y escritas, a cada uno de los ítems a evaluar se le asignó una escala de 0 a 5 , en sentido creciente de nivel demostrado. Como se ha descrito anteriormente, del primer al cuarto ítem corresponden a la misma dimensión, mientras que para el resto, del quinto al undécimo (décimo en caso de habilidades escritas), cada elemento corresponde a una dimensión a evaluar. Esto significa que, en la comunicación oral, había once elementos para cuantificar y ocho dimensiones diferentes de la competencia, mientras que para la comunicación escrita había diez elementos para cuantificar y siete dimensiones. La puntuación global se obtuvo sumando las valoraciones correspondientes de todos los ítems. Por último, para evaluar el nivel de logro de las competencias, se desarrolló una escala de evaluación. El rango de valor total obtenido de las rúbricas de 0 a 55 puntos para la comunicación oral y de 0 a 50 puntos para la comunicación escrita, dividiendo esta en en seis rangos diferentes (Tabla 3) desde nivel bajo a nivel máster.

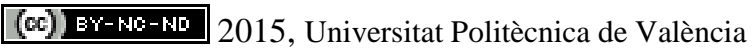


Tabla 3. Nivel de logro de las competencias en comunicación oral y escrita

\begin{tabular}{|c|c|c|c|c|c|}
\hline \multicolumn{7}{|c|}{ ESCALA DE COMUNICACIÓN ORAL } \\
\hline \multirow{2}{*}{ Bajo } & $\begin{array}{c}\text { Bajo- } \\
\text { Medio }\end{array}$ & Medio & Medio-Alto & Alto & Máster \\
\hline de 0 a $<9$ & de 9 a $<17$ & de 17 a $<35$ & de 35 a $<44$ & de 44 a $<50$ & de 50 a 55 \\
\hline \multicolumn{7}{|c|}{ ESCALA DE COMUNICACIÓN ESCRITA } \\
\hline Bajo & $\begin{array}{c}\text { Bajo- } \\
\text { Medio }\end{array}$ & Medio & Medio-Alto & Alto & Máster \\
\hline de 0 a $<8$ & de 8 a $<15$ & de 15 a $<33$ & de 33 a $<40$ & de 40 a $<46$ & de 46 a 50 \\
\hline
\end{tabular}

Estas escalas permiten obtener valores comparables de la competencia de comunicación. Las rúbricas se han desarrollado intentando evaluar diferentes ítems de la comunicación eficaz, delimitando la escala de valoración de la forma más lógica posible, teniendo en cuenta que la mayoría de los estudiantes deberían alcanzar un valor intermedio en el desarrollo de la competencia evaluada. Es decir, el rango de puntuación para los niveles de logro más bajos y más altos serán más reducidos que para los niveles intermedios.

La metodología de evaluación utilizada (basada en rúbricas), ayudó a los autores no sólo a evaluar las habilidades orales, escritas y la competencia de comunicación del alumnado, sino también a determinar en qué medida se han entendido las lecciones, proporcionando una valiosa información acerca del conocimiento por parte de los estudiantes pemitiendo retroalimentar a los alumnos en caso necesario.

Con el objeto de obtener información de la metodología desarrollada (feedback) a los alumnos de Grado, por ser el grupo más numeroso, se les pasó una encuesta de opinión con nueve cuestiones, que se muestra en la Tabla 4. 
Propuesta de evaluación de las competencias de comunicación oral y escrita en asignaturas de Ingeniería Mecánica y Ciencia de Materiales

Tabla 4. Encuesta de opinión al alumnado

1- Indicar los guiones de prácticas que has leído previamente a asistir a la sesión de prácticas, disponibles en PoliformaT|previos a la realización de la práctica:

\begin{tabular}{|c|l|l|l|l|l|}
\hline & Práctica 1 & Práctica 2 & Práctica 3 & Práctica 4 & Práctica 5 \\
\hline Sí & & & & & \\
\hline No & & & & & \\
\hline
\end{tabular}

2- La metodología utilizada en las sesiones de prácticas basada en la preparación de presentaciones orales y su exposición por parte de un integrante del grupo, ha conseguido que mi nivel de motivación de cara a la sesión haya sido (1: Muy baja - 10: Muy alta):

\begin{tabular}{|l|l|l|l|l|l|l|l|l|l|}
\hline 1 & 2 & 3 & 4 & 5 & 6 & 7 & 8 & 9 & 10 \\
\hline & & & & & & & & & \\
\hline
\end{tabular}

3- Consideras interesante de cara a tu futuro profesional la utilización de la metodología empleada de cara a tener la oportunidad de poner en práctica tu capacidad de comunicación oral, así como la de resolver posibles dudas a tus compañeros (1: Muy poco interesante - 10: Muy interesante):

\begin{tabular}{|l|l|l|l|l|l|l|l|l|l|}
\hline 1 & 2 & 3 & 4 & 5 & 6 & 7 & 8 & 9 & 10 \\
\hline & & & & & & & & & \\
\hline
\end{tabular}

4- Valora el interés de la metodología utilizada teniendo en cuenta su objetivo: estructuración y síntesis de la información, asimilación de contenidos, motivación, contribución a una comunicación oral más clara y eficaz, análisis y valoración de los resultados (1: Muy poco interesante - 10: Muy interesante):

\begin{tabular}{|l|l|l|l|l|l|l|l|l|l|}
\hline 1 & 2 & 3 & 4 & 5 & 6 & 7 & 8 & 9 & 10 \\
\hline & & & & & & & & & \\
\hline
\end{tabular}

5- Indicar el porcentaje de conocimiento, según tu opinión, que has alcanzado sobre los contenidos trabajados en la práctica con la preparación del trabajo derivado de la metodología utilizada:

\begin{tabular}{|l|l|l|l|l|l|l|l|l|l|}
\hline $10 \%$ & $20 \%$ & $30 \%$ & $40 \%$ & $50 \%$ & $60 \%$ & $70 \%$ & $80 \%$ & $90 \%$ & $100 \%$ \\
\hline & & & & & & & & & \\
\hline
\end{tabular}

6- Valora a nivel personal la metodología utilizada desde el punto de vista de lo útil (1: Nada útil - 10: Muy útil) que te ha sido en la preparación y asimilación de los contenidos vistos en la sesión de prácticas:

\begin{tabular}{|l|l|l|l|l|l|l|l|l|l|}
\hline 1 & 2 & 3 & 4 & 5 & 6 & 7 & 8 & 9 & 10 \\
\hline & & & & & & & & & \\
\hline
\end{tabular}

(cc) EY-NC-ND 2015, Universitat Politècnica de València

Congreso IN-RED (2015) 
Tabla 4. Encuesta de opinión al alumnado (continuación)

\begin{abstract}
7- De los aspectos que pretendía fomentar en el alumnado la actividad realizada, clasifícalos del más destacable a menos de 1 (Más destacable) al 5 (Menos destacable):
\end{abstract}

\begin{tabular}{|l|l|}
\hline & Valoración \\
\hline Estructuración y síntesis de la información & \\
\hline Asimilación de contenidos & \\
\hline Motivación & \\
\hline Contribución a una comunicación oral más clara y eficaz & \\
\hline Análisis y valoración de los resultados & \\
\hline
\end{tabular}

8- Tras la experiencia realizada con esta metodología, ¿cuál sería tu predisposición a realizar otras sesiones de prácticas usando esta metodología? (1: Completamente en desacuerdo (Nula) - 10: Completamente de acuerdo (Muy adecuada)

\begin{tabular}{|l|l|l|l|l|l|l|l|l|l|}
\hline 1 & 2 & 3 & 4 & 5 & 6 & 7 & 8 & 9 & 10 \\
\hline & & & & & & & & & \\
\hline
\end{tabular}

9- Indica la utilidad de la plantilla de evaluación empleada en la valoración de la exposición realizada por los compañeros (1: Muy baja - 10: Muy alta):

\begin{tabular}{|l|l|l|l|l|l|l|l|l|l|}
\hline 1 & 2 & 3 & 4 & 5 & 6 & 7 & 8 & 9 & 10 \\
\hline & & & & & & & & & \\
\hline
\end{tabular}

\title{
4. Resultados
}

Uno de los objetivos del trabajo, es llevar a cabo una metodología que permita valorar el nivel de logro en la comunicación eficaz, para ello será fundamental evaluar las diferencias de valoración entre los distintos evaluadores, profesores de un lado y profesores y alumnos de otro lado. Estos resultados están directamente relacionados con la eficacia y la fiabilidad de los métodos de evaluación desarrollados. La Figura 1 y Figura 2 muestran una comparación de los datos medios recogidos en en las asignaturas de Grado y Máster, respectivamente, para evaluar la competencia de comunicación oral.

En las Figuras 1a / 2a se comparan las notas medias de las valoraciones de los alumnos, con las media entre los dos profesores para los diferentes actos de evaluación llevados a cabo. Las Figuras 1b / 2b comparan las valoraciones de cada profesor en cada acto de evaluación mostrándose también el promedio y la desviación estándard. Como se esperaba, la valoración de los profesores, en términos generales, fue inferior a la de los alumnos (Figuras 1a / 2a). Sin embargo, y a pesar de las diferencias, existe una aceptable correlación entre las calificaciones de alumnos y profesores. La desviación estándar (DS) en las valoraciones de los alumnos (Figura 1a), no es demasiado alta, ya que el $42 \%$ de los valores son DS $\leq \pm 3$,

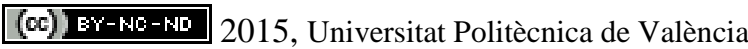


Propuesta de evaluación de las competencias de comunicación oral y escrita en asignaturas de Ingeniería Mecánica y Ciencia de Materiales

mientras que el $63 \%$ se situan con DS $\leq \pm 5$. Comparando entre las valoraciones realizadas por los profesores en un mismo acto de evaluación (Figura 1b), se observa una mayor sintonía (menor dispersión) en las valoraciones obtenidas donde el $74 \%$ de las valoraciones que tienen DS $\leq \pm 3$, mientras que el $95 \%$ de los datos entregando DS $\leq \pm 5$.

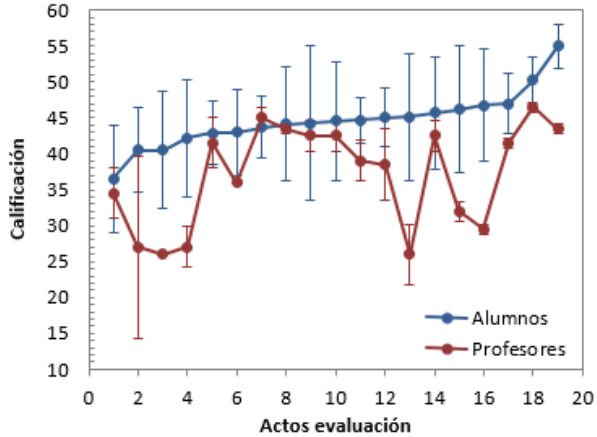

(a)

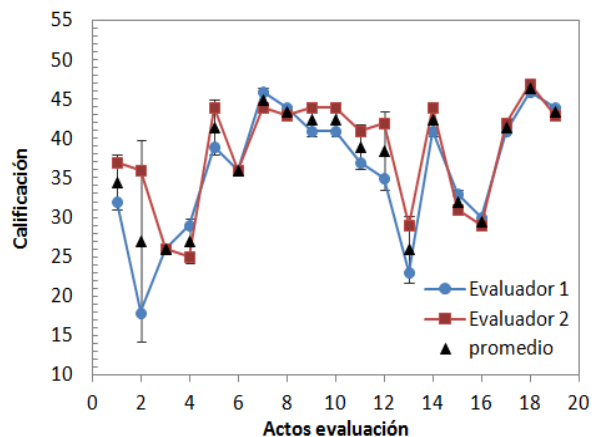

(b)

Fig. 1 Comparativa de los resultados de evaluación de la comunicación oral en los alumnos de Grado: (a) Promedio de la valoración entre alumnos y entre profesores y (b) Valoración de los profesores.

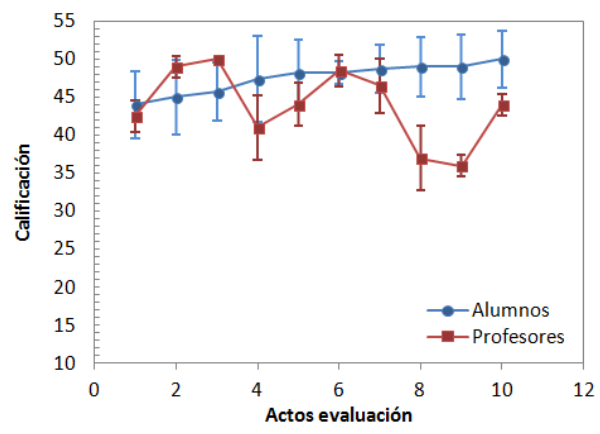

(a)

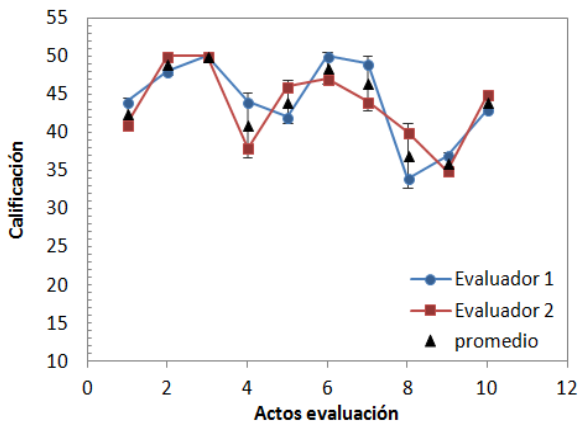

(b)

Fig. 2 Comparativa de los resultados de evaluación de la comunicación oral en los alumnos de Máster:

(a) Promedio de la valoración entre alumnos y entre profesores y (b) Valoración de los profesores

En la asignatura del Máster, se observa un comportamiento similar. En el caso de los alumnos de Máster (Figura 2a) la dispersión disminuye respecto a la obtenida en los alumnos de Grado, obteniendo valores donde el el 60 \% y el 80 \% de las valoraciones proporcionan DS $\leq \pm 3$ y DS $\leq \pm 5$, respectivamente. En el caso de los profesores en la asignatura del Máster (Figura 2b) el 100\% de las valoraciones presenta DS $\leq \pm 5$. Por lo tanto, teniendo en cuenta la influencia de la desviación estándar en las calificaciones finales de los alumnos, éstos supondrían una variación en la calificación final baja, comprendida entre un 5 \% y un 
$9 \%$, teniendo en cuenta los valores de desviación estándar considerados DS $\leq \pm 3$ o DS $\leq \pm 5$, respectivamente. Las Figuras $1 \mathrm{~b} / 2 \mathrm{~b}$, muestran una buena correlación entre las evaluaciones realizadas por dos profesores distintos tanto en Grado como en Máster, lo que ha sido confirmado mediante análisis estadístico con el Test t-Student’s. Este test se utiliza para evaluar si entre dos medias se puede considerar que hay diferencias estadísticamente significativas. En las evaluaciones de los profesores de Grado y Máster, el p-valor obtenido ha sido 0,33 y 0,83 , respectivamente. Al ser superiores al 0,05 , indica la ausencia de diferencia, y por tanto se puede considerar que no hay diferencias estadísticas significativas entre las valoraciones. En el caso de comparar entre estudiantes y profesores en el Grado, se obtuvo que el $53 \%$ de los actos de evaluación no muestran diferencia estadísticamente significativa entre los valores de los alumnos y los profesores. Este valor se eleva hasta el 80\% en el caso de la asignatura del Máster. Este incremento puede deberse a una mayor formación por parte de los alumnos de Máster, así como a la realización de las valoraciones de una forma más objetiva.

El uso de rúbricas ha demostrado ser muy beneficioso en el aprendizaje y la motivación de los estudiantes. La Figura 3 muestra la evaluación de las habilidades de comunicación escrita (estudiantes Máster) en el principio y en el final del curso. La tendencia general en las calificaciones finales globales (Figura 3a), obtenidas a partir rúbrica mostrada en la Tabla 2, es una mejora durante el transcurso del curso. En el caso de analizar cada ítem de forma individual, se observa un aumento en las calificaciones al final de la asignatura, lo que apunta a un mejor desarrollo de la tarea con el tiempo, en definitiva con la formación.

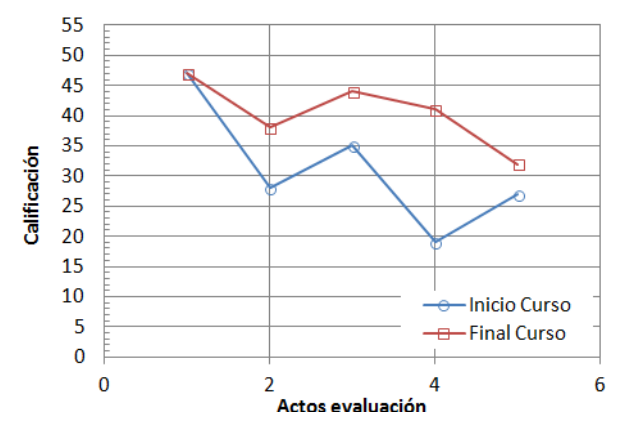

(a)

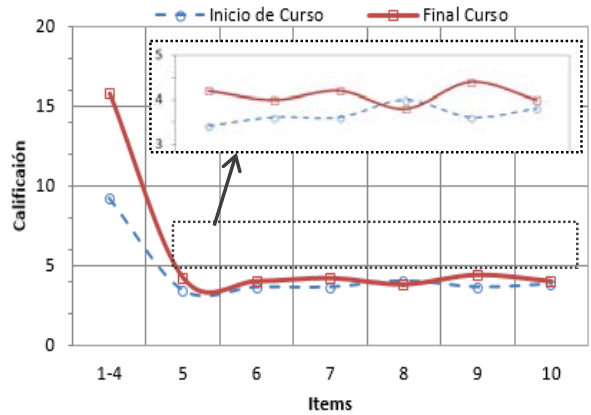

(b)

Fig. 3 Evolución de la evaluación de la competencia comunicación escrita en los alumnos de Máster: (a) Calificación de los alumnos al principio y final del curso y (b) Promedio de las valoraciones obtenidas por ítem evaluado al principio y al final del curso. 
Propuesta de evaluación de las competencias de comunicación oral y escrita en asignaturas de Ingeniería Mecánica y Ciencia de Materiales

En la Figura 4 se muestra una comparación del nivel de consecución o logro en comunicación oral para el alumnado de Grado y Máster, con el propósito de evaluar la flexibilidad de la metodología propuesta.

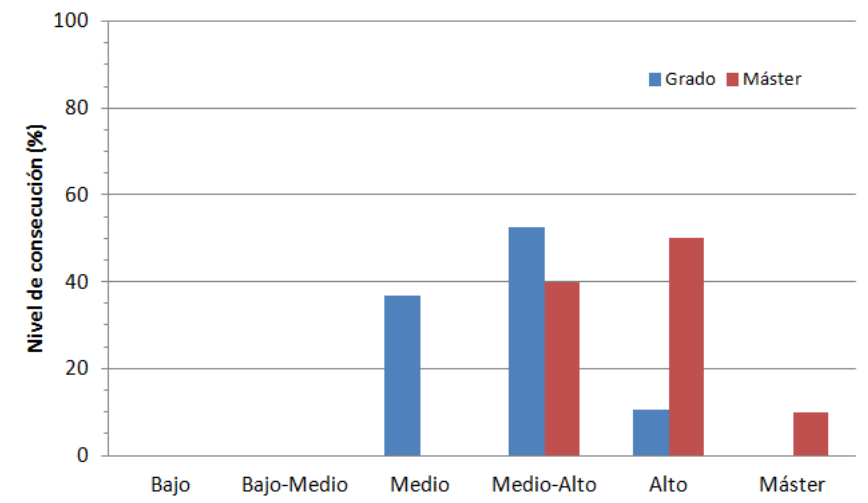

Fig. 4 Comparación del nivel de consecución de la competencia de comunicación oral en estudiantes de Grado y Máster teniendo en cuenta la valoración de los profesores.

Como era previsible, las valoraciones globales son más altas en estudiantes de Máster que en estudiantes de Grado. La distribución de los datos de acuerdo con la escala desarrollada para el nivel de consecución (Tabla 3, escala de comunicación oral), el 37\% de las calificaciones de los estudiantes de Grado están en un nivel medio, mientras que la mayoría de los estudiantes (53\%) se encuentran en un nivel medio-alto y sólo el 10\% de las valoraciones alcanzan un nivel alto en el desarrollo de esta competencia. En el caso de las valoraciones de los alumnos de Máster, el 40\% se encuentra en un nivel medio-alto, del 50\% en un nivel alto y el 10\% de los estudiantes alcanza el nivel Máster. Los resultados coinciden con el nivel de los alumnos, lo que refleja la buena conexión entre la metodología desarrollada y la respuesta obtenida con el instrumento de evaluación empleado (rúbrica) en términos de fiabilidad.

Finalmente, la encuesta de opinión (Tabla 4) realizada sobre la metodología desarrollada para la valoración de la competencia comunicación oral, nos reporta información de lo que piensan los alumnos al respecto. De la primera cuestión, determinamos que únicamente el 14\% de los alumnos de Grado han leído todos los guiones de prácticas de forma previa a asistir a la sesión, mientras que un 18\% no han leído ninguno de estos guiones. La medida de alumnos que han leído el guión por sesión de prácticas es de un 46\%, porcentaje bajo teniendo en cuenta que la evaluación seguida en la asignatura es contínua. En la Tabla 5, se resumen los resultados obtenidos en el resto de cuestiones, mostrando el porcentaje de alumnos con 
valoraciones por encima de 5 y entre 7 y 10 (notable-sobresaliente) en aspecto relacionados con la metodología trabajada.

Tabla 5. Resultados de la encuesta de opinión al alumnado

\begin{tabular}{ccc}
\hline Cuestión & $\begin{array}{c}\text { Valoración }>\mathbf{5} \\
\mathbf{( \% )}\end{array}$ & $\begin{array}{c}\text { Valoración 7 } \mathbf{x} \leq \mathbf{1 0} \\
\mathbf{( \% )}\end{array}$ \\
\hline 2 & 71,4 & 44,0 \\
3 & 91,7 & 79,8 \\
4 & 86,9 & 59,5 \\
5 & 84,5 & 63,1 \\
6 & 83,3 & 59,5 \\
8 & 71,4 & 44,0 \\
9 & 73,8 & 41,7 \\
\hline
\end{tabular}

Como resultado positivo se tiene que, de forma general, a todas las cuestiones planteadas la valoración superior a 5 está por encima del $70 \%$, alcanzando incluso en alguna cuestión valores cercanos al 92\%. La cuestión 3, relacionada con el interés de la metodogía en su futuro profesional, entendida como práctica de comunicación oral, es la que ha obtenido calificaciones más altas. Seguida de la cuestión 4, en la que se valora el interés de la metodología en virtud de los objetivos con la que se planteó. En la cuestión 2, un 44\% asigna una valoración notable-sobresaliente a la motivación conseguida de cara a la sesión de prácticas con la preparación del trabajo. Un resultado altamente satisfactorio es el que reporta la cuestión 5, donde el $63.1 \%$ de los alumnos, indica que ha alcanzado un nivel de conocimiento entre el 70 y el 100\% con la preparación del trabajo. En concordancia con lo anterior, en la cuestión 6, el 60\% de los alumnos valora con una calificación entre 7 y 10 (notable-sobresaliente), la utilidad de la preparación en la asimilación de los contenidos vistos en prácticas. En cuanto a las cuestiones 8 y 9, se obtienen valoraciones superiores al $70 \%$, a la cuestiones de predisposición a usar esta metodología y a la utilidad de la rúbrica para la valoración, respectivamente. Finalmente, en la cuestión 7, atendiendo a los resultados de los alumnos, los aspectos que pretendía fomentar la metodología empleada los han clasificado de la siguiente manera (de más destacable a menos): 1) asimilación de contenidos, 2) estructura y síntesis de la información, 3) motivación y 4) contribución a comunicación oral más clara; eficaz y análisis y valoración de los resultados.

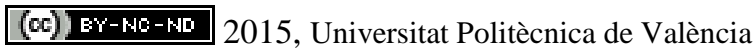

Congreso In-Red (2015) 
Propuesta de evaluación de las competencias de comunicación oral y escrita en asignaturas de Ingeniería Mecánica y Ciencia de Materiales

\section{Conclusiones}

El objetivo de este trabajo es presentar la metodología desarrrollada para la evaluación de la habilidad de comunicación oral y escrita mediante un método basado en rúbricas, realizado en estudiantes de grado y de máster. La metodología seguida para trabajar las competencias es activa y participativa para el alumnado, haciéndoles partícipes además de la evaluación. La disponibilidad de la rúbrica de evaluación desde el inicio, permite a los alumnos conocer qué es lo que tienen que hacer, qué aspectos son importantes y porqué lo están haciendo, promoviendo así su aprendizaje. El análisis de los resultados obtenidos busca comprobar la fiabilidad y la validez de los métodos propuestos.

La buena correlación entre las valoraciones realizadas por los estudiantes y los profesores, confirman la correcta comprensión de los criterios de evaluación utilizados, criterios fáciles de entender y claros para los diferentes evaluadores (profesores, estudiantes de máster y de grado). Por tanto, la metodología propuesta se piensa que se podría utilizar con independencia del marco de la asignatura (asignaturas técnicas) y/o del nivel de estudios universitarios del alumnado. Además, la aplicación de las escalas finales desarrolladas para calificar el nivel de logro de la competencia por parte de los alumnos, confirma la flexibilidad de la metodología ya que los resultados obtenidos en diferentes disciplinas técnicas y niveles pueden ser normalizados y compararados.

Esta metodología presenta una buena correlación de las evaluaciones entre los profesores y profesores-alumnos. En el 80\% de las evaluaciones en el Máster no muestra diferencias estadísticamente significativas entre las valoraciones de profesores-alumnos. La menor correlación obtenida en la asignatura de grado, podrían deberse a la falta de experiencia o entrenamiento del alumnado en la evaluación entre iguales, así como a una dudosa objetividad en la valoración en algunos casos. Otros puntos interesante derivados de la encuesta de opinión son: que los alumnos consideran interesante la metodogía (práctica de comunicación oral) en su futuro profesional, donde el $80 \%$ le da una calificación notablesobresaliente y que más del 70\% tendría una predisposición positiva a continuar utlizando esta metodología.

La experiencia llevada a cabo en la valoración de las competencias por medio de rúbricas promueve el aprendizaje, basándonos en la mejora observada en las calificaciones iniciales y finales de la valoración de la competencia comunicación escrita, y además fomenta el feedback bidireccional.

\section{Agradecimientos}

Los autores agradencen la financiación recibida de la Universitat Politècnica de València a través del proyecto PIME/2014/A/012/B.

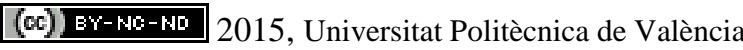

Congreso IN-RED (2015) 


\section{Referencias}

SURSOCK, A., SMIDT, H. (2010). “Trends 2010: A decade of change in european higher education”. Brussels: European University Association.

ANDREWS, J., HIGSON, H. (2008). “Graduate employability, 'Soft skills' versus 'Hard’ business knowledge: A european study”. Higher Education in Europe, 33, 411-422.

DUNBAR, N. E., BROOKS, C. F., KIBICKA-MILLER, T. (2006). “Oral communication skills in higher education: Using a performance-based evaluation rubric to asses communication skills". Innovative Higher Education, 31, 115-128.

ENTWISTLE, N. J., PETERSON, E. R. (2004). “Conceptions of learning and knowledge in higher education: Relationships with study behaviour and influences of learning environments”. International Journal of Educational Research, 41, 407-428.

JONSSON, A., SVINGBY, G. (2007). "The use of scoring rubrics: Reliability, validity and educational consequences”. Educational Research Review, 2, 130-144.

MURIAS, P., de MIGUEL, J. C., RODRIGUEZ, D. (2007). “A composite indicator for university quality assesment: The case of Spanish higher education system”. Social Indicators research, 89, 129146.

RIECKMANN, M. (2012). "Future-oriented higher education: Which key competencies should be fostered through university teaching and learning?”. Futures, 44, 127-135.

SPARKS, J. R., SONG, Y., BRANTLEY, W., LIU, O. L. (2014). “Assessing written communication in higher education: Review and recommendations for next-generation assessment". ETS Research Report Series, 2014, 1-52.

Tuning project. (2014). “Approaches to teaching, learning and assessment in competences based degree programmes”. 